\title{
Structural Study of GaSb, InSb Melts with XAFS Technique
}

\author{
Y.R. Wang, K.Q. Lu and C.X. Li \\ Institute of Physics, Chinese Academy of Sciences, P.O. Box 603-30, Beijing 100080, China
}

\begin{abstract}
The XAFS spectra of GaSb, InSb melts and the corresponding solids are measured at different temperatures. The reverse Monte Carlo simulation technique is applied to the analysis of EXAFS data of molten $\mathrm{GaSb}$, InSb. The partial radial distribution functions of molten $\mathrm{GaSb}$, InSb are given as well as the local atomic structure models which are based on a geometrical analysis of the model atomic configurations. A discussion about the mechanism of semiconductor-metal transition from solid to liquid is presented in the light of the local structure changes
\end{abstract}

\section{INTRODUCTION}

It is well known that GaSb and InSb are two important kinds of semiconductors. It is, however, difficulty to produce the high-quality single crystals of them. Many researches have been done on the properties and structures of GaSb and InSb solids. But there are few studies on the structures of GaSb and InSb liquids due to the difficulties on the experiments. Mizuki reported their results of the neutron diffraction studies on the liquid $\mathrm{GaSb}$. They gave the total pair distribution functions at several temperatures above the melting point of GaSb. Evidently, elucidating the liquid structure is of great help to understand the process of crystal growth. On another hand, the conducting behavior of these two semiconductors transforms from the semiconducting behavior into the metallic one via solid-liquid transition. The structural changes, especially the changes of the local atomic structure, may be related to this semiconductor-metal transition. For example the semiconductor-metal transition occurs across the melting point of Germanium and $a b$ inito molecular dynamic studies [1] on the liquid Germanium showed that several kinds of coordinated polyhedra existed in liquid Germanium other than the unique coordinated tetrahedron in Germanium solid. This was also demonstrated by the neutron diffraction studies [2] on the liquid Germanium.

Although the X-ray and neutron-diffraction techniques are normally used to probe the structural information in liquid systems, the extended X-ray absorption fine structure (EXAFS) technique is a powerfully alternative technique. The more significant point is that the EXAFS signal is sensitive to the local structural changes and can nicely complement the $S(k)$ information [3] derived from the X-ray or neutron - diffraction data. The difficulties of EXAFS studies on liquid systems come from both experiment and data analysis. Recently excellent works have been done [4] to solve these problems.

In this paper we will present our results of EXAFS studies on the GaSb, InSb melts. The paper is arranged as follows. Section 2 contains experimental details on the sample preparation and XAFS measurements. Section 3 contains the description of the data-analysis methods and the results. Section 4 gives the conclusions.

\section{EXPERIMENTAL DETAILS}

\subsection{Sample Preparation}

The key problem in the XAFS experiment for the liquid system is how to keep a uniform thin liquid film vertically during the measurements. To resolve this problem we prepared our XAFS samples in the following way.

Firstly GaSb and LiF fine powders were mixed in some ratio, and then ground adequately in the agate mortar. The mixed powder was pressed to form the disc-like sample with the diameter of $10 \mathrm{~mm}$ and the thickness of $1 \mathrm{~mm}$. Such prepared sample was sealed in the quartz tube and sintered in the oven at $500^{\circ} \mathrm{C}$. The purpose of sintering is to avoid of breaking of the sample in the transfer. Finally we put the sintered sample into the quartz tube as shown in Figure 1. The quartz tube was sealed in the vacuum. The same method was used to prepare the InSb samples.

We know that the melting point of $\mathrm{LiF}$ is higher than that of either GaSb or InSb. When the heating temperature is higher than the melting point of $\mathrm{GaSb}$ or InSb but lower than that of $\mathrm{LiF}, \mathrm{GaSb}$ or InSb melts but LiF does not. This results in the droplets of $\mathrm{GaSb}$ or InSb dispersing uniformly in the solid $\mathrm{LiF}$ support, which is equivalent to keeping a thin liquid film vertically in the measurement. Adding the LiF into the sample can not raise the absorption background seriously because of the low X-ray absorption of $\mathrm{LiF}$. The ratios of $\mathrm{GaSb}$ and $\mathrm{InSb}$ over $\mathrm{LiF}$ can be optimized according to the densities and absorption coefficients of the compounds involved for the appropriate thickness of a sample to satisfy $\mu x \leqslant 1.5$ and $\Delta \mu x \leqslant 1.0$, where $\mu x$ and $\Delta \mu x$ are the total absorption and the absorption jump just above the detected absorption edge. LiF does not react on GaSb and InSb at high temperature and even above the melting points of $\mathrm{GaSb}$ and $\mathrm{InSb}$ through the $\mathrm{X}$-ray diffraction examinations. 


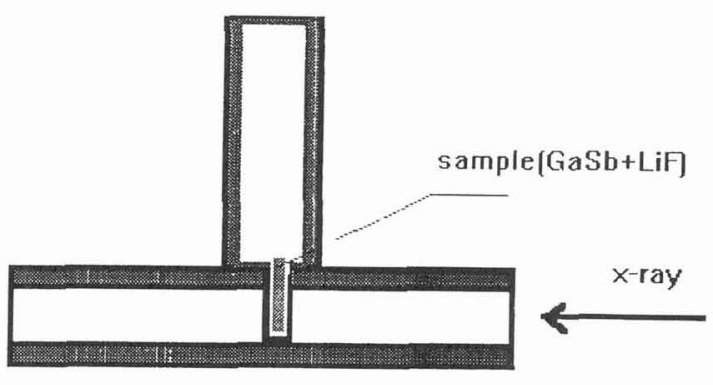

sample holder

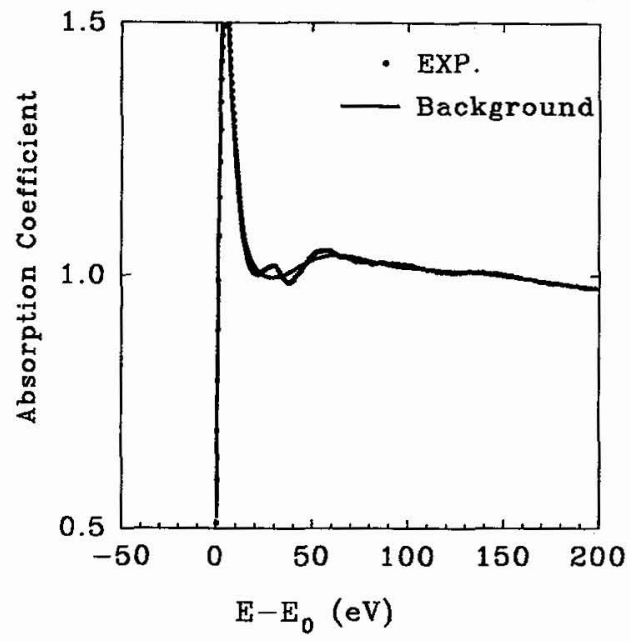

Ga k-edge spectra for GaSb at $720^{\circ} \mathrm{C}$

Figure 2: The raw Ga k-edge absorption spectrum for liquid GaSb at $720^{\circ} \mathrm{C}$ (Solid square) and its background absorption (solid line).

Figure 1: Sample holder.

\subsection{XAFS Experiments}

The XAFS measurements were carried out on the beam line $10 \mathrm{~B}$ at the synchrotron radiation source of the Photon Factory in Japan. A transmission mode was used in the measurement with an $\mathrm{Si}(311)$ channel-cut monochromator. The electron energy in the storage ring was about $2.5 \mathrm{GeV}$ with a current of $200 \mathrm{~mA}$. Two ion chambers filled with a mixture of $\mathrm{Ar}$ and $\mathrm{N}_{2}$ gases were used to detect both incident $\mathrm{X}$-ray intensity and transmitted intensity simultaneously. the Ga-k-edge and Sb- $k$-edge spectra were collected respectively for GaSb samples at room temperature, $500^{\circ} \mathrm{C}, 700^{\circ} \mathrm{C}$ and $720^{\circ} \mathrm{C}$, while In- $k$-edge and Sb-k-edge spectra were measured for InSb samples at room temperature, $485^{\circ} \mathrm{C}$ and $540^{\circ} \mathrm{C}$. For the measurements at high temperatures, the samples was heated in a self-made furnace.

\section{DATA ANALYSIS AND RESULTS}

\subsection{Background Removal}

The first step of the data analysis is the background removal. Because the magnitude of XAFS oscillation for the liquid systems is very small (generally in the order of $10^{-3}$ ), it is very important to remove the background absorption cautiously, otherwise the XAFS oscillation may be distorted seriously. Several methods have been put forward to tackle this problem including the removal of the multielectron excitation signals embedded in the absorption spectra. In this paper we use a new method based on the derivative theorem to remove the background. As an example, Figure 2 gives the raw X-ray absorption spectrum for liquid $\mathrm{GaSb}$ at $720^{\circ} \mathrm{C}$ and its background absorption. The multielectron excitation feature is appeared obviously at $48 \mathrm{eV}$ behind the thresold.

\subsection{RMC Method}

Reverse Monte Carlo (RMC) method [4] was employed to analyze the XAFS signals for the liquid GaSb and InSb. In this paper we just give a simple description about its basic algorithm. For the disordered system the EXAFS signal $\chi_{\alpha}(k)$ can be written as :

$$
\chi_{\alpha}(k)=\sum_{\beta} S_{0}^{2}(k)\left|f_{\beta}(k, \pi)\right| \int_{0}^{\infty} 4 \pi r^{2} \rho_{0} g_{\alpha \beta}(r) \frac{e^{-\frac{2 r}{\lambda}}}{k r^{2}} \sin \left(2 k r+2 \delta_{1}+\varphi_{\beta}\right) \mathrm{d} r
$$

The subscripts $\alpha, \beta$ represent the chemical types of the central and scattering atoms respectively. $g_{\alpha, \beta}(r)$ is the partial radial distribution function. $f_{\beta}(k, \pi)$ is the electron backscattering amplitude and $S_{0}^{2}(k)$ is the amplitude reduction factor which accounts for intrinsic losses and interference. $\lambda$ is the XAFS mean-free path. $\delta_{1}$ is the final state phase shift at the central atom, while $\varphi_{\beta}$ 
stands for the scattering phase shift. We write the contribution to the EXAFS of a single scattering atom at a fixed distance $r$ as $\chi_{1}(k, r)$

$$
\chi_{1}(k, r)=S_{0}^{2}(k)\left|f_{\beta}(k, \pi)\right| \frac{e^{-\frac{2 r}{\lambda}}}{k r^{2}} \sin \left(2 k r+2 \delta_{1}+\varphi_{\beta}\right)
$$

Then the measured XAFS signal can be rewritten as

$$
\chi_{\alpha}(k)=\sum_{\beta} 4 \pi \rho \int_{0}^{\infty} r^{2} g_{\alpha, \beta}(r) \chi_{1}(k, r) \mathrm{d} r
$$

The first step of RMC simulation is to construct an initial configuration. This is a three-dimensional array of $\mathbf{N}$ points, representing atomic coordinates, in a cube of side $L$. The array may be generated at random, or from a lattice of crystal. The only constraint is that it must have the same chemical composition and mass density as the experimental sample. Normal periodic boundary conditions are applied, and then the radial distribution function $g_{s}(r)$ is calculated. A new configuration is generated by random motion of one particle. The new radial distribution function $g_{s}^{\prime}(r)$ is then calculated. From the simulated radial distribution functions $g_{s}(r)$ and $g_{s}^{\prime}(r)$ EXAFS functions $\chi_{s}(k)$ and $\chi_{s}^{\prime}(k)$ are calculated. These two functions are compared with the experimental spectrum from the system which is being modeled. The standard form of comparison is used which is defined as

$$
F I=\sum_{i}\left[k_{i}^{n}\left(\chi_{s}\left(k_{i}\right)-\chi_{\mathrm{E}}\left(k_{i}\right)\right)\right]^{2} / \sigma_{\mathrm{E}}^{2} \sum\left[k_{i}^{n} \chi_{\mathrm{E}}\left(k_{i}\right)\right]^{2}
$$

where $\sigma_{\mathrm{E}}^{2}$ is the estimated squared fractional error on the experimental spectrum. $F I$ and $F I^{\prime}$ are calculated using this equation. If $F i<F i^{\prime}$ then the new configuration is accepted. If $F I>F I^{\prime}$ then the new configuration is accepted with probability $\exp \left[-\left(F I-F I^{\prime}\right)\right]$. If the new configuration is accepted then it becomes the starting configuration, otherwise the old configuration is retained. The process is repeated until $F I$ converges.

\subsection{Results of GaSb}

Crystalline GaSb has the diamond structure with the lattice parameter of $6.096 \AA$. The first near neighbor atoms are at $2.639 \AA$ which compose of a regular tetrahedra. The second shell consists of 12 atoms at $4.3105 \AA$. Figure 3 shows the radial structure functions of GaSb solids at room temperature. It is obvious that only the first shell signal can be distinguished.

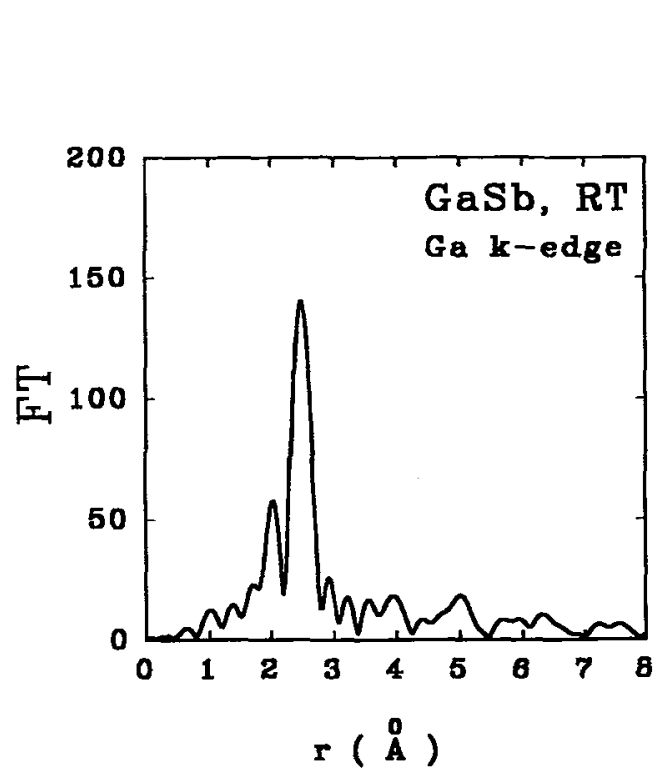

Figure 3: The radial structure function of GaSb solid at room temperature, which is transformed from Ga $k$-edge XAFS spectrum.



$\mathrm{GaSb}, \mathrm{Ga} k$-edge spectra

Figure 4: The $\mathrm{Ga} k$-edge spectra for solid $\mathrm{GaSb}\left(500^{\circ} \mathrm{C}\right.$, solid line $)$ and liquid $\mathrm{GaSb}\left(720^{\circ} \mathrm{C}\right.$, dotted line).

The melting point of GaSb is $709.6^{\circ} \mathrm{C}$. The density of liquid GaSb increases about $8.2 \%$. Figure 4 shows the XAFS oscillation of liquid $\mathrm{GaSb}\left(720^{\circ} \mathrm{C}\right)$ compared with that of the high temperature solid $\left(500^{\circ} \mathrm{C}\right)$. The obvious feature of liquid spectrum is the large amplitude reduction of the XAFS oscillation. This demonstrated the large disorder of liquid system. 
Using the RMC method illustrated in the section 3.2, we analyzed the XAFS data of liquid GaSb. In our RMC simulation 1000 atoms were used, corresponding to a cell length $L=29.6 \AA$. The EXAFS oscillation was fitted out to a distance of $6.0 \AA$, beyond which no discernible feature in the Fourier transform spectrum was observed. The configuration of a perfect GaSb crystal was used as the inital configuration. Ga $k$-edge and $\mathrm{Sb} k$-edge spectra were fitted simultaneously in the simulation. The backscattering amplitudes and phase shifts used to calculate $\chi_{1}(k)$ come from the results of theoretical calculation and experimental data of GaSb solids. Figure 5 shows the Ga-Sb pair distribution function. It can be seen that the first peak is at $2.7 \AA$ ans has a wide distribution range corresponding to the first coordination shell. The information on the $\mathrm{Ga}-\mathrm{Ga}$ and $\mathrm{Sb}-\mathrm{Sb}$ partial radial distribution functions can also be given in the simulation. The EXAFS, however, is only sensitive to the local structure of the absorbing atom. For the large disorder of liquid system, the signal for the intermediate-range distribution is embedded in the noise and can not be distinguished. Thence, the intermediate-range information given by the simulation is not confirmative. Figure 6 shows the RMC fitted and experimental EXAFS functions. It is illustrated that the fitting is well.

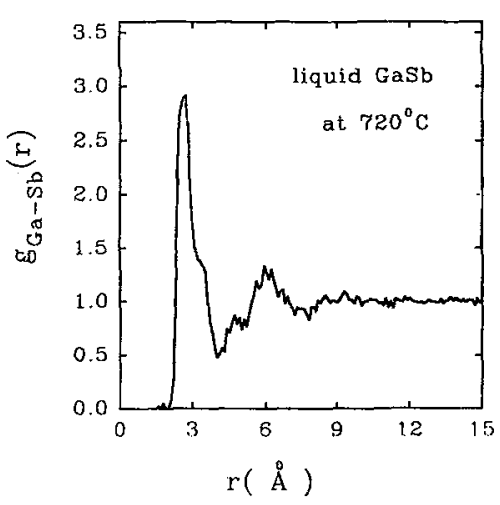

Figure 5: The Ga-Sb pair distribution function for liquid $\mathrm{GaSb}$ at $720^{\circ} \mathrm{C}$.



Figure 6: The RMC fitted and experimental EXAFS oscillations for liquid $\mathrm{GaSb}$ (Ga $k$-edge) at $720^{\circ} \mathrm{C}$.

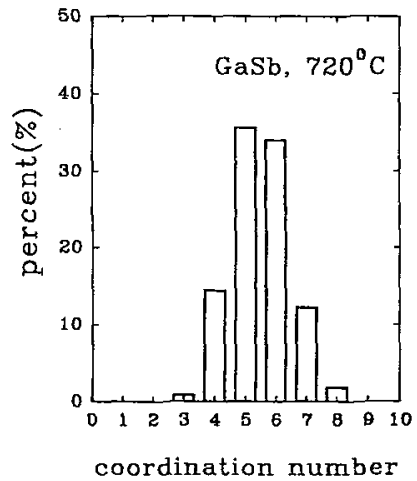

Figure 7: The distribution of the coordination number for the liquid $\mathrm{GaSb}$.

The distribution of the coordination number is calculated according to the generated configuration. To calculate this the maximum of the bond length must be defined, usually the first minimal of the pair distribution function. Figure 7 shows the distribution of the coordination number for the liquid GaSb. From this figure we can see that there are several kinds of coordinated polyhedra in liquid $\mathrm{GaSb}$, other than the unique regular tetrahedra in $\mathrm{GaSb}$ solid. Except for the regular tetrahedra, the coordinated polyhedra can be regarded as geometric defects. The semiconductor-metal transition may be related to the existance of geometric defects. In another aspect the average coordination number is 6.0 , which is smaller than the typical value in liquid metal. So the semiconductor-metal transition is not due to the increase of the coordination number.

\subsection{Results of InSb}

Crystalline InSb has also the diamond structure with the lattice parameter of $6.465 \AA$. The first near neighbor atoms are at $2.805 \AA$ which compose of a regular tetrahedra. The second shell consists of 12 atoms at $4.581 \AA$. The density of liquid InSb increases about $12.5 \%$ compared with that of InSb solid.

The same routine of RMC simulation has been applied to analyze the XAFS data of liquid InSb. Figure 8 shows in In-Sb pair distribution function, while Figure 9 illustrates the RMC fitted and experimental EXAFS functions. Figure 10 gives the distribution of the cowordination number, which also demonstrates the existence of several kinds of polyhedra in liquid InSb.

\section{CONCLUSIONS}

The XAFS spectra of GaSb, InSb melts and the corresponding solids are measured at different temperatures. The reverse Monte Carlo simulation is applied to the analysis of EXAFS data of molten GaSb, InSb. The radial distribution functions are given. The coordination number and its distribution are calculated. 


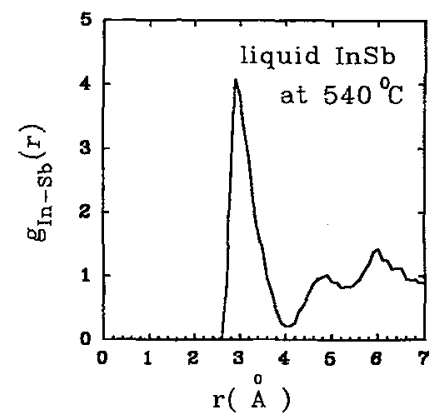

Figure 8: The In-Sb pair distribution function.



Liquid InSb, In $k$-edge, $540^{\circ} \mathrm{C}$

Figure 9: Illustrates the RMC fitted and experimental EXAFS functions.



Figure 10: Gives the distribution of the coordination number.

\section{Acknowledgments}

We would like to thank Mr Xiao Nian and Dr Wen Weijia for their assistance with the experiments. We wish to thank both the Photon Factory of Japan and Synchrotron Radiation Laboratory in Beijing. This work is supported by the Chinese National Science Foundation and a grant for Key Research Project in Climbing Program from the State Science and Technology Commission of China.

\section{References}

[1] Kresse, G. and Hafner, J., Phys. Rev. B 49 (1994) 14251-14269.

[2] Petkov, V. and Yunchov, G., J. Phys. : Cond. Matter 6 (1994) 10885-10895.

[3] Filipponi, A., J. Phys. Cond. Matter. 6 (1994) 8415.

[4] Gurman, J., McGreevy, R.L., J. Phys. : Cond. Matter 2 (1990) 9463-9473. 